\title{
Flutuação populacional de Rotylenchulus reniformis em solo cultivado com algodoeiro
}

\author{
Guilherme Lafourcade Asmus ${ }^{(1)}$ e Cassiana Miki Ishimi(1)
}

(1)Embrapa Agropecuária Oeste, Caixa Postal 661, CEP 79804-970 Dourados, MS. E-mail: asmus@cpao.embrapa.br, cassiana@cpao.embrapa.br

\begin{abstract}
Resumo - O objetivo deste trabalho foi caracterizar a flutuação populacional de nematoide reniforme (Rotylenchulus reniformis) em solo cultivado com algodoeiro, em função de variáveis edafoclimáticas. Em uma área naturalmente infestada - com média de 680 nematoides por $200 \mathrm{~cm}^{3}$ de solo, em Aral Moreira, MS - foi estabelecida uma grade de amostragem com 30 pontos, equidistantes em $50 \mathrm{~m}$. No período de outubro de 2004 a junho de 2006, amostras de solo $(0,0-0,2 \mathrm{~m}$ e $0,2-0,4 \mathrm{~m})$ foram coletadas mensalmente, para avaliação da abundância e composição dos diferentes estágios da população do nematoide. Foram também mensuradas a precipitação, a temperatura e a umidade do solo a 0,15 e $0,30 \mathrm{~m}$. Foi possível observar que a densidade e a composição populacional de $R$. reniformis são influenciadas pela presença de plantas de algodoeiro. Maior abundância desse nematoide foi encontrada à profundidade de $0,2-0,4 \mathrm{~m}$. A precipitação e a umidade do solo pouco influenciaram a população de $R$. reniformis. A temperatura do solo, a 0,15 e $0,30 \mathrm{~m}$ de profundidade, correlaciona-se com a população de $R$. reniformis a $0,2-0,4 \mathrm{~m}$ de profundidade.
\end{abstract}

Termos para indexação: Gossypium hirsutum, anidrobiose, nematoide reniforme, temperatura do solo, umidade do solo.

\section{Population fluctuation of Rotylenchulus reniformis in soil cultivated with cotton}

Abstract - The objective of this work was to determine the population fluctuation of reniform nematodes (Rotylenchulus reniformis) in a cotton production area as affected by soil and climate conditions. A grid of 30 sampling sites, equidistant in $50 \mathrm{~m}$ from each other, was established in a field naturally infested - with an average of 680 reniform nematodes per $200 \mathrm{~cm}^{3}$ soil, in Aral Moreira county, Mato Grosso do Sul, Brazil. Soil samples at $0.0-0.2$ and $0.2-0.4 \mathrm{~m}$ depths were collected monthly on each sampling site, between October 2004 and June 2006, for the evaluation of nematode abundance and population composition. Measurements of soil temperature and moisture at 0.15 and $0.30 \mathrm{~m}$ depth and rainfall were made in each sampling site. It was observed that cotton plants play important roles in the abundance and composition of reniform nematode populations. The abundance of $R$. reniformis was higher at $0.2-0.4 \mathrm{~m}$ depth. Rainfall and soil moisture contents had little influence on reniform nematode population. Soil temperatures at both 0.15 and $0.30 \mathrm{~m}$ were correlated to $R$. reniformis population density at $0.2-0.4 \mathrm{~m}$ depth.

Index terms: Gossypium hirsutum, anhydrobiosis, reniform nematode, soil temperature, soil humidity.

\section{Introdução}

O nematoide reniforme (Rotylenchulus reniformis Linford \& Oliveira, 1940) (Nemata: Hoplolaimidae) constitui-se num dos principais nematoides que parasitam a cultura do algodoeiro (Gossypium hirsutum L.) (Starr, 1998), e está amplamente distribuído nas regiões tropicais e subtropicais (Robinson et al., 1997). No Brasil, embora tenha sido detectado em várias regiões produtoras de algodão (Gielfi et al., 2003; Asmus, 2004; Inomoto, 2006), sua importância como patógeno da cultura não tem sido devidamente considerada, em razão de os sintomas que provoca na parte aérea das plantas serem confundidos com os de outras causas, como compactação do solo e desordens nutricionais. Além disso, $R$. reniformis não causa sintomas visíveis nas raízes (Robinson et al., 1997; Starr, 1998). Ocorre em praticamente todas as áreas de cultivo do algodão, e seu manejo demanda enormes esforços e gastos (Heald \& Robinson, 1990).

Até o momento, não são evidentes os motivos da expansão da área infestada pelo nematoide reniforme. Algumas hipóteses baseiam-se em sua habilidade de competir com outras espécies e de, em condições 
adversas, procurar camadas mais profundas do solo para sobreviver. A adaptação de $R$. reniformis a diferentes texturas de solos permite a esse nematoide explorar ambientes que são inadequados a outros fitonematoides (Thomas \& Clark, 1983). Além dessas hipóteses, sua tolerância ao estresse hídrico, por um mecanismo de anidrobiose - juvenis enovelam-se sobre si e reduzem drasticamente o metabolismo -, também tem sido considerada (Torres et al., 2006).

Informações sobre dinâmica populacional e mecanismos de sobrevivência são essenciais para o manejo integrado de fitonematoides. Tome-se, por exemplo, o desenvolvimento de técnica de manejo de Meloidogyne incognita conhecida como "alqueive úmido" (Dutra \& Campos, 2003), que é baseada no conhecimento de que juvenis de segundo estágio eclodem no período de entressafra, em condições de umidade e temperatura adequadas, mesmo na ausência de hospedeiros favoráveis. De forma semelhante, a prevalência de formas móveis, pré-parasitárias, de $R$. reniformis no solo, durante o período de entressafra, poderia constituir-se em fragilidade do nematoide a estratégias de controle.

O objetivo deste trabalho foi caracterizar a abundância e a composição da população de Rotylenchulus reniformis, em função de variáveis edafoclimáticas.

\section{Material e Métodos}

O estudo foi conduzido em área com aproximadamente 20 hectares, naturalmente infestada por Rotylenchulus reniformis (média de 680 nematoides por $200 \mathrm{~cm}^{3}$ de solo), cultivada há oitos anos com algodoeiro, na fazenda Bom Futuro, Município de Aral Moreira, MS. O solo caracteriza-se como Latossolo Vermelho distroférrico, de textura argilosa (16\% de areia, $9 \%$ de silte e $75 \%$ de argila). Em outubro de 2004, foram estabelecidos 30 pontos de amostragem georreferenciados (Tabela 1), equidistantes $(50 \times 50 \mathrm{~m})$, que constituíram as unidades de avaliação. Nos anos agrícolas 2004/2005 e 2005/2006, foi cultivado o algodoeiro cultivar Delta Opal, em sistema de plantio direto, sobre palhada de centeio (Secale cereale L.). As datas de semeadura do algodoeiro foram 9/11/2004 e 2/11/2005, e as colheitas ocorreram em 12/4/2005 e 4/4/2006, respectivamente. O centeio foi sempre semeado imediatamente após a colheita de algodão e destruição da soqueira com roçadeira.
De outubro de 2004 a junho de 2006, foram coletadas, mensalmente (com exceção de novembro de 2004), amostras de solo de cada ponto de amostragem. Para tal, foram abertas trincheiras a $0,1 \mathrm{~m}$ da linha de plantio do algodoeiro, com $0,4 \mathrm{~m}$ de profundidade, e o solo foi coletado (aproximadamente $1 \mathrm{dm}^{3}$ ) de duas profundidades: $0,0-0,2$ e $0,2-0,4 \mathrm{~m}$. As amostras foram embaladas em saco de plástico, etiquetadas e levadas em caixa térmica ao Laboratório de Nematologia da Embrapa Agropecuária Oeste, em Dourados, MS, para a extração de nematoides, pelo método descrito por Jenkins (1964). Após a extração, os nematoides foram inativados em banho-maria $\left(55^{\circ} \mathrm{C}\right.$ por $\left.5 \mathrm{~min}\right) \mathrm{e}$ fixados em formalina (2\%). Os nematoides presentes em cada amostra foram identificados e quantificados. No caso de $R$. reniformis, foram quantificados, individualmente: juvenis, fêmeas jovens, machos. Nas amostras coletadas a partir de fevereiro de 2005, foram quantificados também os espécimes em estado de anidrobiose. A identificação da espécie foi feita por meio de estudo morfométrico (Robinson et al., 1997).

Tabela 1. Coordenadas dos pontos de amostragem.

\begin{tabular}{|c|c|c|c|}
\hline Ponto & Coordenada & Ponto & Coordenada \\
\hline \multirow[t]{2}{*}{1} & S22 $54^{\prime} 54,1^{\prime \prime}$ & 16 & S22054'54,1'" \\
\hline & W55'31'39, $1^{\prime \prime}$ & & W553ㄴ $31^{\prime} 46,2^{\prime \prime}$ \\
\hline \multirow[t]{2}{*}{2} & $\mathrm{~S} 22^{\circ} 54^{\prime} 54,5^{\prime \prime}$ & 17 & $\mathrm{~S} 22^{\circ} 54^{\prime} 53,5^{\prime \prime}$ \\
\hline & W55'31'40,9' & & W55'31'44,4' \\
\hline \multirow[t]{2}{*}{3} & $\mathrm{~S} 22^{\circ} 54^{\prime} 55,2^{\prime \prime}$ & 18 & $\mathrm{~S} 22^{\circ} 54^{\prime} 52,9^{\prime \prime}$ \\
\hline & W55'31'42,4'" & & W55'31'42,9' \\
\hline \multirow[t]{2}{*}{4} & S22054'55,7'" & 19 & $\mathrm{~S} 22^{\circ} 54^{\prime} 52,4^{\prime \prime}$ \\
\hline & W55'31'44,2'" & & W55'31'41,2' \\
\hline \multirow[t]{2}{*}{5} & $\mathrm{~S} 22^{\circ} 54^{\prime} 56,1^{\prime \prime}$ & 20 & $\mathrm{~S} 22^{\circ} 54^{\prime} 51,0^{\prime \prime}$ \\
\hline & W55'31'45,6"' & & W55'31'39,8' \\
\hline \multirow[t]{2}{*}{6} & $\mathrm{~S} 22^{\circ} 54^{\prime} 56,6^{\prime \prime}$ & 21 & $\mathrm{~S} 22^{\circ} 54^{\prime} 52,1^{\prime \prime}$ \\
\hline & W55'31'47,3' & & W55'31'39,6"' \\
\hline \multirow[t]{2}{*}{7} & $\mathrm{~S} 22^{\circ} 54^{\prime} 57,0^{\prime \prime}$ & 22 & $\mathrm{~S} 22^{\circ} 54^{\prime} 51,2^{\prime \prime}$ \\
\hline & W55'31'49,0"' & & W55 $31^{\prime} 41,8^{\prime \prime}$ \\
\hline \multirow[t]{2}{*}{8} & $\mathrm{~S} 22^{\circ} 54^{\prime} 57,7^{\prime \prime}$ & 23 & $\mathrm{~S} 22^{\circ} 54^{\prime} 51,9^{\prime \prime}$ \\
\hline & W55 $31^{\circ} 50,7^{\prime \prime}$ & & W55 $35^{\circ} 143,2^{\prime \prime}$ \\
\hline \multirow[t]{2}{*}{9} & $\mathrm{~S} 22^{\circ} 54^{\prime} 58,3^{\prime \prime}$ & 24 & $\mathrm{~S} 22^{\circ} 54^{\prime} 52,4^{\prime \prime}$ \\
\hline & W55'31'52,6"' & & W55 $31^{\circ} 45,0^{\prime \prime}$ \\
\hline \multirow[t]{2}{*}{10} & $\mathrm{~S} 22^{\circ} 54^{\prime} 58,6^{\prime \prime}$ & 25 & $\mathrm{~S} 22^{\circ} 54^{\prime} 52,8^{\prime \prime}$ \\
\hline & W55'31'54,1"' & & W55'31'46,3' \\
\hline \multirow[t]{2}{*}{11} & $\mathrm{~S} 22^{\circ} 54^{\prime} 58,4^{\prime \prime}$ & 26 & $\mathrm{~S} 22^{\circ} 54^{\prime} 53,5^{\prime \prime}$ \\
\hline & W55'31'54,0"' & & W553 $31^{\prime} 48,0^{\prime \prime}$ \\
\hline \multirow[t]{2}{*}{12} & S22 $54^{\prime} 56,3^{\prime \prime}$ & 27 & $\mathrm{~S} 22^{\circ} 54^{\prime} 53,9^{\prime \prime}$ \\
\hline & W55'31'52,7'" & & W55'31'49,8' \\
\hline \multirow[t]{2}{*}{13} & $\mathrm{~S} 22^{\circ} 54^{\prime} 55,8^{\prime \prime}$ & 28 & $\mathrm{~S} 22^{\circ} 54^{\prime} 54,6^{\prime \prime}$ \\
\hline & W55'31'51, $1^{\prime \prime}$ & & W55 $31^{\prime} 51,3^{\prime \prime}$ \\
\hline \multirow[t]{2}{*}{14} & $\mathrm{~S} 22^{\circ} 54^{\prime} 55,1^{\prime \prime}$ & 29 & $\mathrm{~S} 22^{\circ} 54^{\prime} 54,6^{\prime \prime}$ \\
\hline & W55'31'49,3'" & & W55'31'51,8' \\
\hline \multirow[t]{2}{*}{15} & $\mathrm{~S} 22^{\circ} 54^{\prime} 54,8^{\prime \prime}$ & 30 & $\mathrm{~S} 22^{\circ} 54^{\prime} 55,5^{\prime \prime}$ \\
\hline & W55 $31^{\prime} 47,8^{\prime \prime}$ & & W55 $35^{\circ} 54,6^{\prime \prime}$ \\
\hline
\end{tabular}


Em cada dia de coleta, foram realizadas medições da temperatura e umidade do solo nas duas profundidades, de cada um dos pontos de amostragem, entre 8 e $11 \mathrm{~h}$. Para as mensurações de temperatura, foi utilizado um geotermômetro digital Soloterm 1200, com sondas de 0,15 e $0,30 \mathrm{~m}$ de profundidade. As sondas foram introduzidas no solo e esperou-se, em média, 5 min até a estabilização da temperatura. Em fevereiro, março e abril de 2005, foram mensuradas as temperaturas apenas na profundidade de $0,15 \mathrm{~m}$, e não foram feitas as medições em maio de 2006. A umidade do solo foi determinada pelo método gravimétrico. A precipitação pluvial foi medida diariamente, com um pluviômetro instalado a cerca de $1.000 \mathrm{~m}$ da área experimental. Os dados foram submetidos a análises de correlação de Pearson, entre as diferentes variáveis mensuradas, tendo-se utilizado o aplicativo SAEG (Ribeiro Júnior, 2001). Para comparações de médias, utilizou-se o teste $t$ de Student.

\section{Resultados e Discussão}

Os valores médios da população de $R$. reniformis estão apresentados na Figura 1. A população do nematoide no solo variou de acordo com a presença ou ausência da cultura do algodoeiro. De modo geral, a população aumentou após a semeadura do algodoeiro, permaneceu alta durante o período em que a cultura esteve no campo (média de 1.102 nematoides por $200 \mathrm{~cm}^{3}$ de solo), e declinou após a colheita. Durante a entressafra do algodoeiro - período em que o solo foi cultivado com centeio, cultura não hospedeira -, a população de $R$. reniformis permaneceu com valores médios de 581 nematoides por $200 \mathrm{~cm}^{3}$ de solo, ou seja, $52,5 \%$ da quantidade observada durante o período em que o algodoeiro estava no campo. O menor número de nematoides foi observado no mês de agosto de 2005, e os maiores, em fevereiro de 2005 e abril de 2006.

Foram verificadas diferenças nos padrões de desenvolvimento da população de $R$. reniformis nas duas safras de algodoeiro. Em 2005, observou-se um claro pico da população em fevereiro, quando a cultura estava em plena floração. Em 2006, foram observados dois picos populacionais: em janeiro (início de floração) e abril (colheita). A baixa densidade populacional observada em março de 2006 não seguiu o padrão esperado, e indica que houve efeito também de outros fatores, que não a presença do hospedeiro.
$\mathrm{O}$ aumento populacional de $R$. reniformis no solo está de acordo com o observado por Norton (1989) e McSorley (1998), de que a planta hospedeira exerce forte efeito sobre a dinâmica da população de nematoides.

$\mathrm{Na}$ média dos 20 meses de observação, a população de $R$. reniformis, na profundidade de $0,2-0,4 \mathrm{~m}\left(1.603 \pm 65\right.$ nematoides por $\left.200 \mathrm{~cm}^{3}\right)$, foi significativamente maior $(p<0,01)$ do que na profundidade de $0,0-0,2 \mathrm{~m}(797 \pm 53$ nematoides por $\left.200 \mathrm{~cm}^{3}\right)$. As únicas exceções ocorreram nos meses de janeiro e abril de 2006. Westphal \& Smart (2003) analisaram a densidade populacional de $R$. reniformis $\mathrm{e}$ a umidade do solo nas profundidades de $0-0,3,0,3-0,6$, 0,6-0,9 e 0,9-1,2 m, durante três safras agrícolas, nas culturas de algodão, milho e sorgo, no estado do Texas, EUA, e observaram que as densidades populacionais, em geral, foram maiores nas camadas mais profundas. Robinson et al. (2005) observaram que, em 15 de 17 campos de algodoeiro de seis estados produtores dos EUA, a população de $R$. reniformis foi superior nas profundidades maiores que $0,30 \mathrm{~m}$, que é normalmente a profundidade de aração e de amostragem para análise nematológica naquele país. Esse resultado pode explicar o aumento populacional do nematoide verificado em áreas de algodoeiro no Brasil (Asmus \& Inomoto, 2007), já que as amostragens, normalmente realizadas no perfil de $0-0,2 \mathrm{~m}$, podem ter subestimado o nematoide presente em áreas de produção, e a população foi detectada apenas quando os danos à cultura já eram visíveis.

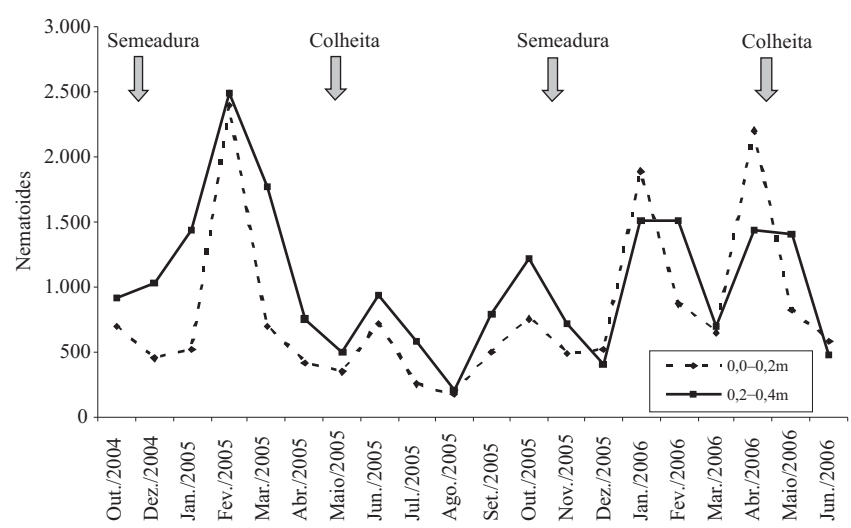

Figura 1. Flutuação populacional de Rotylenchulus reniformis no solo (número de nematoides por $200 \mathrm{~cm}^{3} \mathrm{de}$ solo), em duas safras de algodoeiro, nas profundidades de $0,0-0,2$ e $0,2-0,4 \mathrm{~m}$. 
A presença do algodoeiro no campo também exerceu evidente influência sobre os diferentes estágios de R. reniformis (Figura 2). A partir da metade da estação de cultivo, prevaleceram formas juvenis, o que ficou especialmente comprovado no final do ano agrícola 2004/2005. Esta observação segue a lógica de que,
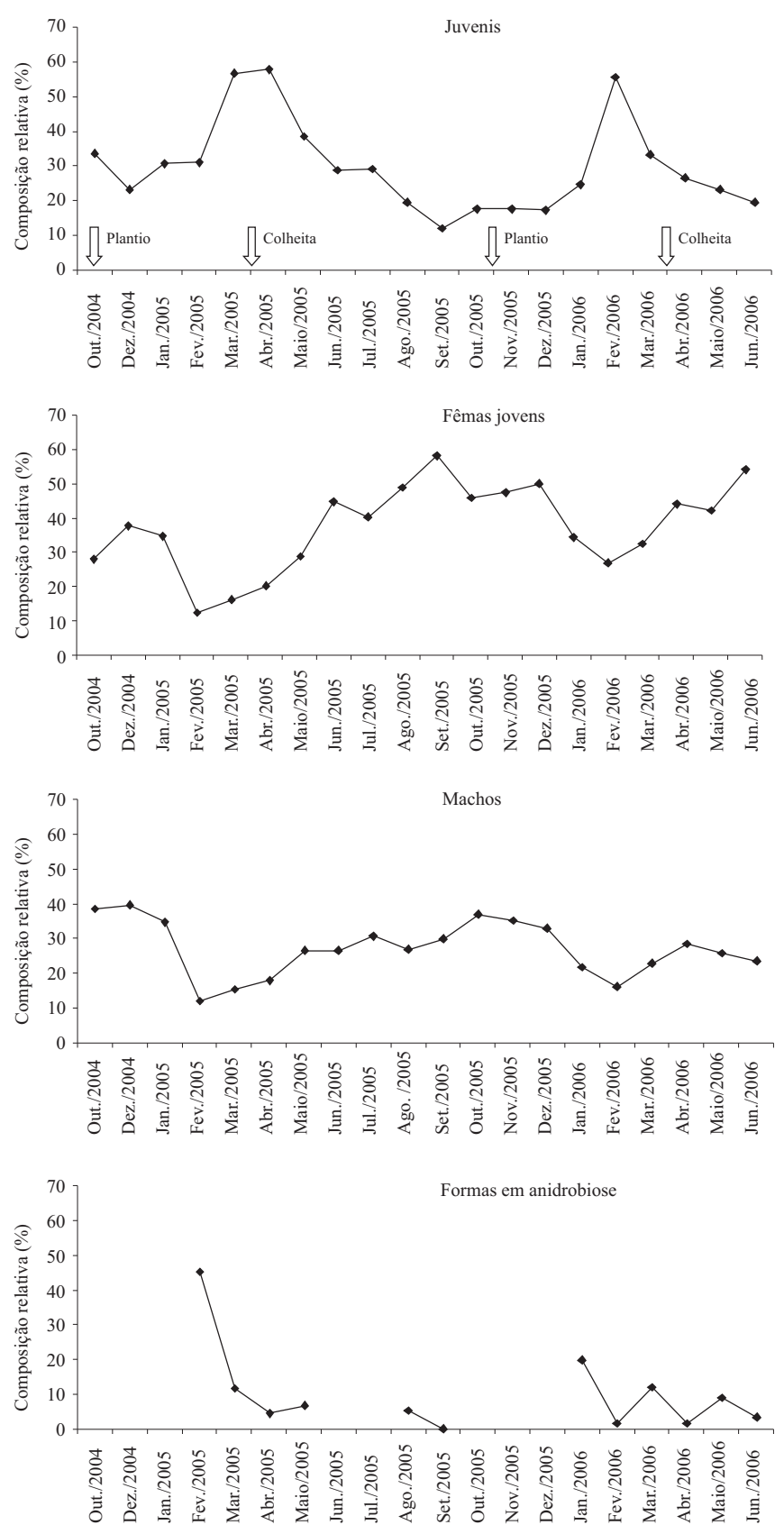

Figura 2. Composição relativa de juvenis, fêmeas jovens, machos e formas em anidrobiose da população de Rotylenchulus reniformis no solo, durante 20 meses de amostragem em área de produção de algodão. durante a fase inicial da cultura, o nematoide multiplicase intensamente e produz grande número de ovos, dos quais eclodem as formas juvenis de segundo estágio que, durante o período de aproximadamente 10 dias, sofrem sucessivas ecdises (Robinson, 2002) e dão origem a grande número de formas juvenis, de terceiro e quarto estágio, o que resulta no grande número de formas jovens da metade para o final do ciclo do algodoeiro. Com a colheita e a consequente escassez de raízes suscetíveis ao parasitismo, novas formas juvenis deixam de ser produzidas e as existentes dão origem a fêmeas e machos, que passam a predominar na população. As populações de fêmeas jovens (imaturas) e de machos seguiram padrões muito semelhantes entre si, com diminuição da densidade durante a safra e aumento durante a entressafra do algodoeiro. Considerando-se que a única fase infectiva é a de fêmea jovem (Robinson, 2002), provavelmente, com a presença do hospedeiro, essas formas migram do solo para as raízes e, assim, têm sua abundância diminuída no solo, em termos proporcionais. Fato semelhante ocorre com o nematoide-de-galhas do algodoeiro, M. incognita, cujos juvenis de segundo estágio - a única forma infectiva - são observados em pequeno número no solo, em presença de plantas de algodoeiro, em razão da rápida penetração nas raízes após a eclosão (Starr, 1998). Um aspecto que chama a atenção é o relativo baixo número de machos em relação ao de fêmeas ( 27 e $37,2 \%$ da população, respectivamente, na média dos 20 meses), o que contraria o conhecimento prévio (Robinson et al., 1997) de que a última ecdise de $R$. reniformis dá origem a aproximadamente a mesma proporção de fêmeas vermiformes parasitárias (fêmeas jovens) e machos não parasitos.

A quantificação de nematoides em anidrobiose foi realizada em apenas 12 dos 20 meses em que se realizou o presente estudo. Diferentemente do esperado, a ausência do hospedeiro e a menor umidade ocorrida durante a entressafra aparentemente não resultaram em maior proporção de nematoides em anidrobiose no solo. Nos dois anos agrícolas, observou-se a ocorrência de anidrobiose a partir da segunda metade do ciclo da cultura do algodoeiro, que coincidiu com a menor umidade do solo, porém com clara diminuição a partir da colheita. A anidrobiose é uma importante estratégia utilizada por diferentes espécies de nematoides, para sobreviver a condições adversas, notadamente a baixos teores de umidade do solo (Womersley \& Ching, 1989). No caso de R. reniformis, a baixa umidade do 
solo estimula formas jovens a se enovelarem, para sobreviver à desidratação. Após o restabelecimento da umidade, emergem como formas pré-adultas (Womersley \& Ching, 1989). A análise do coeficiente de correlação de Pearson (r) mostrou haver correlação negativa significativa entre a umidade do solo a $0,2-0,4 \mathrm{~m}$ e o número de nematoides em anidrobiose a $0,0-0,2 \mathrm{~m}(\mathrm{r}=-0,1149 ; \mathrm{p}<0,01)$ e a $0,2-0,4 \mathrm{~m}$ $(r=-0,0992 ; p<0,01)$. Porém, os baixos coeficientes de correlação evidenciam que outros fatores, além da umidade, podem influenciar a ocorrência de nematoides nessa condição.

A precipitação mensal, durante o período do estudo, variou de $20 \mathrm{~mm}$ (fevereiro de 2005) a $299 \mathrm{~mm}$ (outubro de 2005) (Figura 3). Entretanto, não se detectou correlação significativa entre a chuva acumulada nos 30 dias que antecederam às avaliações e as densidades populacionais do nematoide, em ambas as profundidades.

A umidade do solo variou de $18,8 \%$ (fevereiro de 2006 ) a $28,3 \%$ (outubro de 2004), na profundidade de $0,15 \mathrm{~m}$, e de $22,7 \%$ (março de 2005 ) a $30,3 \%$ (outubro de 2004), na profundidade de $0,30 \mathrm{~m}$ (Figura 4). A umidade do solo não exerceu efeito significativo sobre a população de $R$. reniformis no solo. Não se observou correlação significativa entre a população do nematoide, nas profundidades de $0,0-0,2$ e $0,2-0,4 \mathrm{~m}$, e a umidade do solo nas profundidades de $0,15 \mathrm{~m} \mathrm{e}$ $0,30 \mathrm{~m}$.

Westphal \& Smart (2003) não observaram efeito da umidade do solo e da profundidade das raízes sobre a população do nematoide no campo. Rebois (1973b) avaliou a infectividade e o desenvolvimento

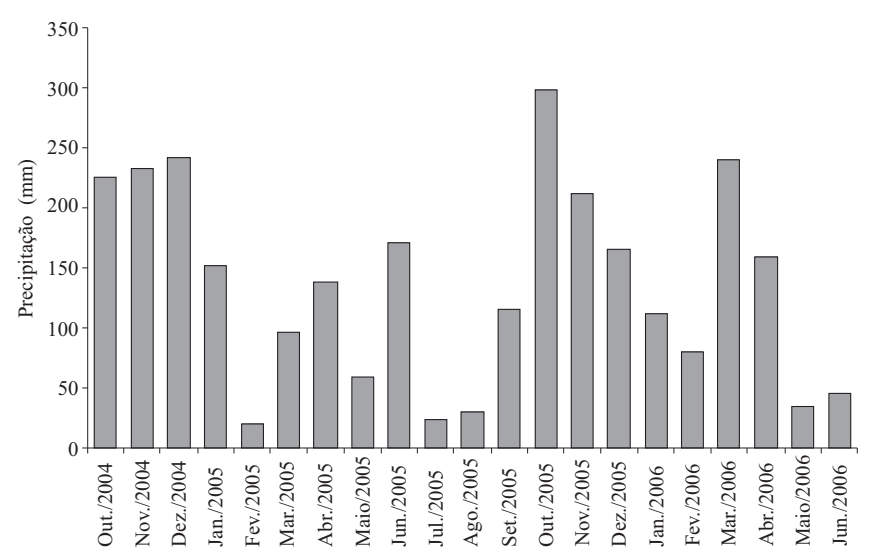

Figura 3. Precipitação mensal ocorrida durante o período do estudo. de $R$. reniformis em soja (Glycine max L.), cultivada em vasos com solo com umidade entre 3,4 e 19\%, em condições controladas, e observou que umidades próximas à capacidade de campo e entre 3,4 e 5,8\% foram prejudiciais ao nematoide, no que se refere a essas duas variáveis. Em campo, quando a umidade a $0,1 \mathrm{~m}$ de profundidade, de solo cultivado com bananeira, variou entre 18 e $35 \%$, durante avaliações mensais realizadas ao longo de um ano, Gantait et al. (2006) observaram correlação significativa e positiva $(\mathrm{r}=0,821)$ entre a umidade e a densidade populacional de $R$. reniformis, na profundidade de $0,0-0,2 \mathrm{~m}$. Diferentemente deste trabalho, em que a presença da cultura anual suscetível (algodoeiro) exerceu visível efeito sobre a flutuação populacional do nematoide, Gantait et al. (2006) trabalharam com uma cultura perene (bananeira), em que a presença do hospedeiro foi constante durante o período de avaliação.
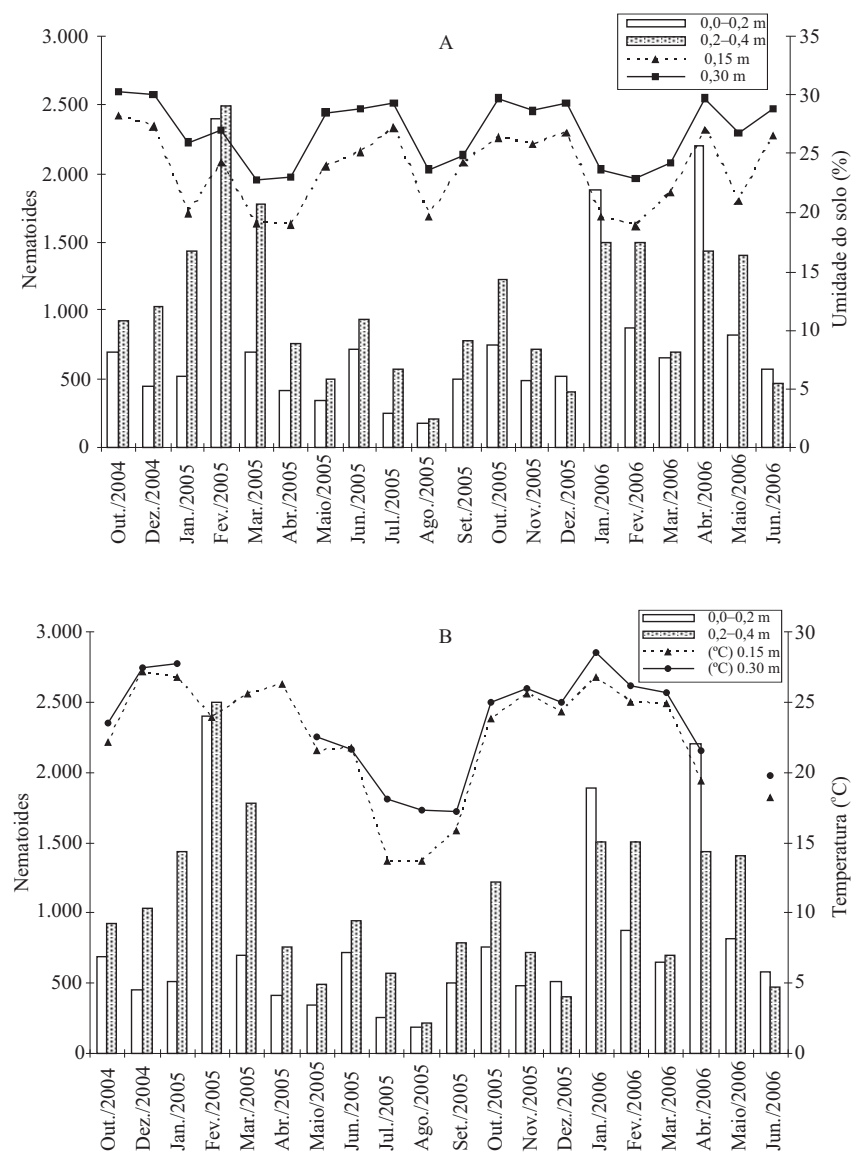

Figura 4. Flutuação da população de Rotylenchulus reniformis (número de nematoides por $200 \mathrm{~cm}^{3}$ de solo) em função da umidade (A) e da temperatura (B) do solo, em duas profundidades do perfil. 
Quando a presença de plantas hospedeiras não é limitante, é possível que os limites de umidade observados possam exercer maior influência sobre a densidade populacional do nematoide. É possível, ainda, que o tipo do solo possa interferir no efeito da umidade sobre o nematoide. A textura argilosa do solo, no presente trabalho, com alta capacidade de retenção de água, pode ter condicionado mudanças menos drásticas na disponibilidade hídrica entre as avaliações, o que teria exercido um menor impacto sobre $R$. reniformis (Sehgal \& Gaur, 1989).

A temperatura do solo variou entre $13,6^{\circ} \mathrm{C}$ (julho de 2005) e $27,2^{\circ} \mathrm{C}$ (dezembro de 2004) na profundidade de $0,0-0,2 \mathrm{~m}$, e entre $17,2^{\circ} \mathrm{C}$ (agosto de 2005) e $27,7^{\circ} \mathrm{C}$ (janeiro de 2005) na profundidade de 0,2-0,4 m. Nesses limites, observou-se correlação significativa entre a densidade populacional de $R$. reniformis, na profundidade de $0,2-0,4 \mathrm{~m}$, e a temperatura do solo a $0,15 \mathrm{~m}(\mathrm{r}=0,4594 ; \mathrm{p}=0,0239)$ e $0,30 \mathrm{~m}(\mathrm{r}=0,5118$; $\mathrm{p}=0,00256)$. Na Figura 4, são apresentados os dados da flutuação da população do nematoide e da temperatura, nas duas profundidades do perfil.

De acordo com Heald \& Inserra (1988), essa espécie apresenta maior taxa de penetração, reprodução e sobrevivência em temperaturas médias entre $25 \mathrm{e}$ $35^{\circ} \mathrm{C}$. Solos secos e temperatura abaixo de $25^{\circ} \mathrm{C}$ causam rápida redução da população do nematoide no solo (Heald \& Inserra, 1988). Ao estudar o efeito das temperaturas do solo de $15,21,5,25,29,5$ e $36^{\circ} \mathrm{C}$ sobre $R$. reniformis, na cultura da soja em casa de vegetação, Rebois (1973a) observou que o melhor desenvolvimento do nematoide ocorreu a 25 e $29,5^{\circ} \mathrm{C}$. Nesse mesmo trabalho, não houve reprodução do nematoide a 15 ou $36^{\circ} \mathrm{C}$. Em condições de campo, Ismail \& El-Nadgi (2005) observaram correlação entre a temperatura do solo e a densidade populacional de $R$. reniformis associada a uma espécie de jasmim (Jasminum grandiflorum L.) e de pinheiro-de-natal [Araucaria excelsa (Lamb.) R. Br]. Gantait et al. (2006) também observaram correlação positiva $(r=0,463)$ entre a densidade populacional desse nematoide e a temperatura do solo a $0,1 \mathrm{~m}$ de profundidade, em cultivo de bananeira, quando a temperatura variou entre 17 e $35^{\circ} \mathrm{C}$. De maneira geral, as temperaturas ocorridas durante o período do presente estudo foram aparentemente adequadas ao desenvolvimento de $R$. reniformis, exceto nos meses de julho, agosto e setembro de 2005, quando foram mais baixas, o que coincidiu com a redução da população do nematoide no solo. Embora provenientes de análise de correlação, os dados obtidos no presente estudo confirmam, em condições de campo, os trabalhos de Rebois (1973a) e Heald \& Inserra (1988).

Durante o período do estudo, foram quantificados os demais nematoides presentes no solo, agrupados em diferentes grupos tróficos: bacteriófagos, micófagos, predadores e outros fitoparasitos. Observou-se correlação positiva entre a população de $R$. reniformis e a de nematoides predadores $(\mathrm{r}=0,3491 ; \mathrm{p}=0,01)$. É possível que o aumento da população do nematoide reniforme tenha selecionado a população de inimigos naturais previamente presentes no solo, representados pelos nematoides predadores. Esse resultado indica a possibilidade de que o controle natural desse nematoide pode ser estimulado por meio de práticas adequadas de manejo, que aumentem a atividade biológica do solo. Dentro do grupo dos nematoides fitoparasitos, além de $R$. reniformis, a espécie mais abundante foi Scutellonema brachyurus Andrassy (com população média entre 1,25 e 647,25 nematoides por $200 \mathrm{~cm}^{3}$ de solo). Observou-se antagonismo entre $R$. reniformis e $S$. brachyurus, que se mostraram inversamente correlacionados $(r=-0,3077 ; p=0,0001)$ nos diversos pontos de amostragem, ao longo dos 20 meses do estudo. A alta capacidade de competição de $R$. reniformis com outras espécies de nematoides foi relatada por López (1987) e Baird et al. (1996). Contudo, pelos dados obtidos no presente trabalho, não há como afirmar se $S$. brachyurus ocorre em baixa densidade populacional em razão da competição exercida por $R$. reniformis ou se acontece o contrário.

\section{Conclusões}

1. A densidade populacional e a composição da população de Rotylenchulus reniformis são influenciadas pela presença de plantas de algodoeiro na lavoura.

2. Solos de áreas de produção de algodão, com suspeita de ocorrência de $R$. reniformis, devem ser preferencialmente amostrados na profundidade de $0,2-0,4 \mathrm{~m}$.

3. A precipitação e a umidade do solo não exercem influência sobre a população de $R$. reniformis.

4. A abundância da população de $R$. reniformis é influenciada pela temperatura do solo. 


\section{Agradecimentos}

À Fundação de Apoio ao Desenvolvimento do Ensino, Ciência e Tecnologia do Estado de Mato Grosso do Sul e ao Conselho Nacional de Desenvolvimento Científico e Tecnológico, pelo auxílio financeiro; ao Engenheiro Agrônomo Karl Isenberg, por ceder a área para a realização do trabalho.

\section{Referências}

ASMUS, G.L. Ocorrência de nematóides fitoparasitos em algodoeiro no Estado de Mato Grosso do Sul. Nematologia Brasileira, v.28, p.77-86, 2004.

ASMUS, G.L.; INOMOTO, M.M. Manejo de nematóides. In: FREIRE, E.C. (Ed.). Algodão no Cerrado do Brasil. Brasília: Abrapa, 2007. p.551-580.

BAIRD, R.E.; DAVIS, R.F.; ALT, P.J.; MULLINIX, B.G.; PADGETT, G.B. Frequency and geographical distribution of plantparasitic nematodes on cotton in Georgia. Journal of Nematology, v.28, p.661-667, 1996.

DUTRA, M.R.; CAMPOS, V.P. Manejo do solo e da irrigação como nova tática de controle de Meloidogyne incognita em feijoeiro. Fitopatologia Brasileira, v.28, p.608-614, 2003.

GANTAIT, V.V.; BHATTACHARYA, T.; CHATTERJEE, A. Fluctuation of nematode populations associated with banana plantation in Medinipur District, West Bengal, India. Indian Journal of Nematology, v.36, p.223-225, 2006.

GIELFI, F.S.; SANTOS, J.M.; ATHAÍDE, M.L.F. Reconhecimento das espécies de fitonematóides associadas ao algodoeiro (Gossypium hirsutum L.) no Estado de Goiás. In: CONGRESSO BRASILEIRO DE ALGODÃO, 4., 2003, Goiânia. Algodão: um mercado em evolução: anais. Campina Grande: Embrapa Algodão; Goiânia: Fundação GO, 2003. (Embrapa Algodão. Documentos, 118). 1 CD-ROM.

HEALD, C.M.; INSERRA, R.N. Effect of temperature on infection and survival of Rotylenchulus reniformis. Journal of Nematology, v.20, p.356-361, 1988.

HEALD, C.M.; ROBINSON, A.F. Survey of current distribution of Rotylenchulus reniformis in the United States. Journal of Nematology, v.22, p.695-699, 1990.

INOMOTO, M.M. Nematóides e seu controle. In: MORESCO, E. (Ed.). Algodão: pesquisas e resultados para o campo. Cuiabá: Facual, 2006. p.241-260.

ISMAIL, A.E.; EL-NADGI, W.M.A. Season population fluctuations of plant parasitic and other nematodes associated with Jasminum grandiflorum and Araucaria excelsa in relation to soil temperature in Giza, Egypt. Pakistan Journal of Nematology, v.23, p.141-148, 2005.

JENKINS, W.R. A rapid centrifugal-flotation technique for separating nematodes from soil. Plant Disease Reporter, v.48, p.692, 1964.
LÓPEZ, G.J. Fluctuación estacional de la distribución espacial de Meloidogyne incognita y Rotylenchulus reniformis en papaya (Carica papaya L.). Turrialba, v.37, p.165-170, 1987.

McSORLEY, R. Population dynamics. In: BARKER, K.R.; PEDERSON, G.A.; WINDHAM, G.L. (Ed.). Plant and nematode interactions. Madison: American Society of Agronomy, 1998. p.109-134.

NORTON, D.C. Abiotic soil factors and plant-parasitic nematode communities. Journal of Nematolgy, v.21, p.299-307, 1989.

REBOIS, R.V.Effect of soil temperature on infectivity and development of Rotylenchulus reniformis on resistant and susceptible soybeans, Glycine max. Journal of Nematology, v.5, p.10-13, 1973a.

REBOIS, R.V. Effect of soil water on infectivity and development of Rotylenchulus reniformis on soybean, Glycine max. Journal of Nematology, v.5, p.246-249, 1973 b.

RIBEIRO JÚNIOR, J.I. Análises estatísticas no SAEG. Viçosa: DPI/UFV, 2001. 301p.

ROBINSON, A.F. Reniform nematodes: Rotylenchulus species. In: STARR, J.L.; COOK, R.; BRIDGE, J. (Ed.). Plant resistance to parasitic nematodes. Wallingford: CABI, 2002. p.153-174.

ROBINSON, A.F.; AKRIDGE, R.; BRADFORD, J.M.; COOK, C.G.; GAZAWAY, W.S.; KIRKPATRICK, T.L.; LAWRENCE, G.W.; LEE, G.; McGAWLEY, E.C.; OVERSTREET, C.; PADGETT, B.; RODRÍGUEZ-KÁBANA, R.; WESTPHAL, A.; YOUNG, L.D. Vertical distribution of Rotylenchulus reniformis in cotton fields. Journal of Nematology, v.37, p.265-271, 2005.

ROBINSON, A.F.; INSERRA, R.N.; CASWELL-CHEN, E.P.; VOVLAS, N.; TROCCOLI, A. Rotylenchulus species: identification, distribution, host ranges, and crop plant resistance. Nematropica, v.27, p.127-180, 1997.

SEHGAL, M.; GAUR, H.S. Effect of the rate of soil moisture loss on the survival, infectivity and the development of Rotylenchulus reniformis, the reniform nematode. Pakistan Journal of Nematology, v.7, p.83-90, 1989.

STARR, J.L. Cotton. In: BARKER, K.R.; PEDERSON, G.A.; WINDHAM, G.L. (Ed.). Plant and nematode interactions. Madison: American Society of Agronomy, 1998. p.359-379.

THOMAS, R.J.; CLARK, C.A. Effects of concomitant development on reproduction of Meloidogyne incognita and Rotylenchulus reniformis on sweet potato. Journal of Nematology, v.15, p.215-220, 1983.

TORRES, G.R.C.; PEDROSA, E.M.R.; MOURA, R.M. Sobrevivência de Rotylenchulus reniformis em solo naturalmente infestado submetido a diferentes períodos de armazenamento. Fitopatologia Brasileira, v.31, p.203-206, 2006.

WESTPHAL, A.; SMART, J.R. Depth distribution of Rotylenchulus reniformis under different tillage and crop sequence systems. Phytopathology, v.93, p.1182-1189, 2003.

WOMERSLEY, C.; CHING, C. Natural dehydration regimes as a prerequisite for the successful induction of anhydrobiosis in the nematode Rotylenchulus reniformis. Journal of Experimental Biology, v.143, p.359-372, 1989.

Recebido em 11 de outubro de 2008 e aprovado em 30 de dezembro de 2008 